\title{
8. TRACE ELEMENT DETERMINATIONS BY X-RAY FLUORESCENCE ANALYSIS: ADVANTAGES, LIMITATIONS, AND ALTERNATIVES ${ }^{1}$
}

\author{
Kurt Boström² and Wolfgang Bach ${ }^{3}$
}

\begin{abstract}
A statistical study of data files obtained by repeated X-ray fluorescence analyses (XRF) of many major and 13 trace components in 20 reference materials was conducted aboard JOIDES Resolution. The results indicate that XRF is excellent for determining most main elements in many rocks and of some trace elements (for instance $\mathrm{Nb}, \mathrm{Y}, \mathrm{Sr}$ and $\mathrm{Rb}$ ) even at low concentrations (4-10 ppm) and that XRF data obtained aboard ship are of the same quality as XRF data reported from land-based laboratories.

Other trace components, however, are harder to determine by XRF; thus, Ba concentrations below $20 \mathrm{ppm}$ in basaltic rocks are impossible to measure with satisfactory precision under routine conditions, and determinations of $\mathrm{Ce}, \mathrm{V}$, and $\mathrm{Zr}$ at low levels may be of questionable quality.

Additional trace element data can be obtained through alternative, more versatile methods, which will permit a significant broadening of the geochemical programs aboard JOIDES Resolution.
\end{abstract}

\section{INTRODUCTION}

Most chemical rock analyses conducted during Ocean Drilling Program (ODP) cruises are made by X-ray fluorescence spectrometry (XRF). This technique has distinct advantages compared with some other methods for determining major and some trace components in rocks. The preparation of samples for XRF analysis is generally simple and requires no knowledge of wet-chemistry techniques or other chemical complications (Bertin, 1975), and many instrument models are rugged and well suited for field or shipboard operations. Furthermore, a study of the counting statistics (Willard et al., 1988) shows that XRF data, when needed, may be further improved by prolonged counting times.

The XRF method is also advantageous in that it yields major element data that agree favorably with published consensus values (referred to as "best values," "recommended values," etc.) for various standard rocks and minerals. Thus, results from ODP Legs 135 and 136 suggest relative errors of about $1 \%$ for $\mathrm{Si}, \mathrm{Ti}, \mathrm{Fe}, \mathrm{Ca}$, and $\mathrm{K}$, and $3 \%-5 \%$ for $\mathrm{Al}, \mathrm{Mn}, \mathrm{Na}$, and $\mathrm{P}$ for the standard rock BHVO-1 (Shipboard Scientific Party, 1992a, table 3; Hergt and Sims, 1994), in good agreement with results observed in our laboratories or reported elsewhere. Similar observations were made during ODP Leg 142 (see the "Explanatory Notes" chapter, tables 2 and 3, in Storms, Batiza, et al., 1993). For instance, the $\mathrm{SiO}_{2}$ data in these tables show deviations of $0.66(=2 \sigma)$ from the calibration curve based on the standard samples and a precision error for BHVO-1 of $0.22(=2 \sigma)$.

\footnotetext{
${ }^{1}$ Batiza, R., Storms, M.A., and Allan, J.F. (Eds.), 1995. Proc. ODP, Sci. Results, 142: College Station, TX (Ocean Drilling Program).

${ }^{2}$ Department of Geology and Geochemistry, Stockholm University, 10691 Stockholm, Sweden.

${ }^{3}$ Institut für Geowissenschaften und Lithosphärenforschung, Justus-Liebig Universität, 6300 Giessen, Federal Republic of Germany. (Present address: Geo Forschungs Zentrum Potsdam, Projektbereich 4.2, Telegrafenberg A50, 14473 Potsdam, Federal Republic of Germany.)
}

The square of the total error $\mathrm{E}_{\mathrm{T}}$ for a determination is equal to the sum of the squares for the contributing errors $E_{1}, E_{2}, E_{3} \ldots$ etc. (see Hunt and Wilson [1986]); that is

$$
\mathrm{E}_{\mathrm{T}}^{2}=\mathrm{E}_{1}^{2}+\mathrm{E}_{2}^{2}+\mathrm{E}_{3}^{2} \ldots \ldots+\mathrm{E}_{\mathrm{n}}^{2} \text {. }
$$

Using the errors in the silica determinations given above we thus find

$$
\mathrm{E}_{\mathrm{T}}^{2}=(0.66)^{2}+(0.22)^{2},
$$

that is, the 2 sigma total random error $\mathrm{E}_{\mathrm{T}}$ for $\mathrm{SiO}_{2}=0.70$. (The additional errors are probably of little significance).

This result implies that $95 \%$ of all $\mathrm{SiO}_{2}$-determinations will have relative errors less than $\pm 1.4 \% \mathrm{SiO}_{2}$ at the $50 \%$ level. However, this estimate is probably too high. A closer study of all data for both goniometers in table 2 ("Explanatory notes" chapter, Storms, Batiza, et al., 1993) reveals that the errors are a curved function of the $\mathrm{SiO}_{2}$ content. For the interval of interest here, $45 \%-55 \% \mathrm{SiO}_{2}$, we find one standard deviation from the regression $(\mathrm{Sy} \cdot \mathrm{x}$ ) to equal 0.23 . (Crow et al., 1960; Snedecor and Cochran, 1967). This value yields a total random error $(2 \sigma)$ of 0.51 and a relative error of about $1 \%$ for $\mathrm{SiO}_{2}$ at the $50 \%$ level, using the same calculations as above. This result is in excellent agreement with the error suggested for $\mathrm{SiO}_{2}$ by Shipboard Scientific Party (1992a). However, round-robin studies (Govindaraju, 1987) show that it is more difficult to define good recommended values for silica than for some other constituents (e.g., $\mathrm{Al}_{2} \mathrm{O}_{3}, \mathrm{Fe}_{2} \mathrm{O}_{3 \mathrm{~T}}$, $\mathrm{MnO}, \mathrm{CaO}, \mathrm{K}_{2} \mathrm{O}$, and $\mathrm{TiO}_{2}$ ). These problems are partly related to clustering of data because of selected methods and may be considerable for some determinations (Abbey, 1991), which will affect all calibration curves based on such geostandards. Useful guides for the statistical test of data for geostandards have been presented by Lister (1982; 1985).

XRF appears to be less well suited for the determination of some trace components in rocks. We will discuss these limitations at some length below and add a brief discussion of alternative analytical ship- 
board methods, which would permit a significantly broader geochemical program at sea.

Until recently, the reliability of the XRF method for trace determinations has been little studied by ODP, judging from the unrealistic detection limits implied in many Initial Reports of the Ocean Drilling Program, as shown below. Using data for 20 geostandards available aboard JOIDES Resolution, we will show that the XRF method yields good data for many trace elements, but poor determinations for others. The results support the findings by Hergt and Sims (1994), who studied these reproducibilities using two geostandards. A preliminary study of these problems is presented in table 3 in the "Explanatory Notes" chapter (Storms, Batiza, et al., 1993), in which precisions for trace element determinations in BHVO-1 are reported. A signal minus background value that is more than 2-3 times larger than one standard deviation in the element signal (SD) for various elements is generally considered indicative of a good detection limit, which thus could be about $18-27 \mathrm{ppm}$ for $\mathrm{Ba}, 8-12 \mathrm{ppm}$ for $\mathrm{Ce}$, and about 1-1.5 ppm for $\mathrm{Cu}$. However, the definition of detection limits is beset with many problems, one of the more troubling ones being that both the studied signal and its background have standard deviations. This has led some authors to advocate the concept limit of determination as as better indicator of reasonable values (Hunt and Wilson, 1986; Moore, 1989).

A discussion of "good" and "bad" data requires an understanding of the accuracies involved, which in theory are impossible to know. A common assumption, though, is that the use of various standard materials will permit a reasonably good estimate of the accuracies. The various merits of the terms "best values," "consensus values," or "recommended values," etc., and how to find the best values for these standard materials are discussed at great length in Flanagan (1986) and Abbey (1988); hereinafter we will simply use the term recommended value (RV). However, recommended values do not represent the ultimate truth, but can change considerably with time (Gladney and Roelandts, 1988; Govindaraju, 1984, 1987, 1989a, 1989b). Continued comparative studies are therefore a must, because they make us aware of how much we still have to learn about many analytical problems.

An indiscriminate pursuit of ultimate accuracies when such results are not needed is indeed meaningless (Skoog and West, 1982). However, studies of the distribution, gain, and loss of $\mathrm{Ba}, \mathrm{Cu}, \mathrm{Ni}$, and other trace constituents because of hydrothermal alterations require very good data both for the leached and the conservative constituents (Rona et al., 1980; Arvanitides et al., 1990). Also, some of these elements (e.g., $\mathrm{Ti}, \mathrm{Zr}, \mathrm{Ba}, \mathrm{Ce}, \mathrm{Rb}$, and $\mathrm{Nb}$ ) are of great interest for petrochemical characterizations of rocks. However, discussions of analytical errors are surprisingly often misunderstood, as was obvious after Fairbairn (1951) published studies of these problems. Some even consider it "unfair" to compare data obtained in different laboratories and under various circumstances; no doubt such studies sometimes are mistakenly considered as personal attacks. However, such tests must be done if we want to understand how reliable data from different laboratories are and if the data can be used in the same study, as was also emphasized by Dick, Erzinger, Stokking, et al. (1992), and Hergt and Sims (1994). Furthermore, it is sometimes believed that the manufacturer's performance report for a specific instrument can replace such tests, but few if any serious analysts support this optimistic attitude (Hunt and Wilson, 1986).

\section{RELIABILITY OF XRF FOR TRACE ELEMENT DETERMINATIONS}

\section{Instrumentation and Experimental Procedures}

Most instrumental and procedural aspects are given in the "Explanatory Notes" chapter of the Initial Reports volume (Storms, Ba- tiza, et al., 1992) for Leg 142, but the following points merit emphasis.

The XRF unit on the ship is an Applied Research Laboratories 8420 wavelength-dispersive spectrometer, employing a 3-kW rhodium target X-ray tube as excitation source. The laboratories aboard ship also contain a hydraulic rock crusher, in which rocks are kept in sturdy plastic bags and liners during crushing to minimize contamination. The grinders are puck and disc mills (sometimes also referred to as swing mills, disc grinders, or barrels), with grinding surfaces of sintered alumina or tungsten carbide. The alumina-lined grinders were used extensively on Leg 142, whereas tungsten carbide liners were used on previous legs. Most grinding procedures involve some contamination problems, but these are small for alumina vials, unless they crack (see Boström and Bach, this volume). The finely ground sample powders are pressed into flat pellets at a standardized pressure and then analyzed.

\section{Statistical Study of Shipboard Data}

To test the quality of XRF determinations aboard JOIDES Resolution we made a statistical study of 1580 measurements of 13 trace elements in 20 different geostandards. These elements and standards are listed in Tables 1 and 2. All data were available during Leg 142 in an open data file in the ship's XRF facility. Most values were obtained on the ship during ODP Legs 140 and 142, but some had been determined at Stanford University. This data file also contains 152 measurements that lack corresponding recommended values in the literature; these values are therefore not considered further. None of the analysts are specified in the XRF file. Furthermore, many elements are present in very low concentrations, primarily $\mathrm{Nb}, \mathrm{Y}, \mathrm{Cu}$, $\mathrm{Ni}$, and $\mathrm{Ce}$, which has caused gaps in the data, because no determination was possible. The only element with data for all geostandards is $\mathrm{Zn}$.

For most trace components in each geostandard there is a set of about six to eight determinations. Using these values arithmetic means were calculated and are presented as found values (FV) in Table 1; this Table also contains the associated standard deviations (SD, $1 \sigma$ ) and corresponding recommended values (RV). A major compilation of recommended values has been presented by Govindaraju (1989b). Table 1 is the data source for all graphs in Figures 1 to 3.

For an initial principal discussion we will study the data for the elements $\mathrm{Cu}, \mathrm{Ba}, \mathrm{Y}$, and $\mathrm{Sr}$, which are representative of the variations in analytical quality of all the trace elements. Plots of FV vs. RV reveal that the XRF data yield good results for high concentrations of the analytes, as can be seen in Figure 1. In some of these plots the highest values have been deleted to better resolve the low-concentration data. The correlation coefficients for the regression lines in the graphs are all near 1.0 (see Fig. 1 caption), but this does not indicate which elements are best determined at low levels. Thus, the data for $\mathrm{Ba}$ are better correlated than data for $\mathrm{Y}$, yet the detection limit for $\mathrm{Ba}$ is much poorer than that for $\mathrm{Y}$, as will be shown below. This is because the correlation coefficients are more controlled by the total spread in the values than by scatter at low levels.

Copper is generally considered to be easy to determine by XRF. For high concentrations this is obvious (Fig. 1A) but the reproducibilities and accuracies are poorer at low concentrations, as will be shown below.

Figure $2 \mathrm{~A}$ shows the relations between $\mathrm{SD}$ and $\mathrm{FV}$ for $\mathrm{Cu}$ in a loglog diagram; linear scales produce hyperbolic curve relations that are hard to analyze over several orders of magnitude. The advantages of logarithmic plots for data analyses were emphasized by Ahrens (Ahrens and Taylor, 1961). Using the regression line for Cu in Figure 2A, we find that the relative percentage standard deviation for FV (= $\mathrm{SD} / \mathrm{FV}$ ) of $10 \%$ is at the $25-\mathrm{ppm}$ level, and at 8 -ppm Cu the corresponding relative error is $30 \%$, implying that a $\mathrm{Cu}$ value below $8 \mathrm{ppm}$ is risky to use. 
Table 1. Geostandards used for precision and accuracy studies of XRF data, and corresponding recommended values.

\begin{tabular}{|c|c|c|c|c|c|c|c|c|c|c|c|c|c|c|c|c|c|c|c|c|}
\hline $\begin{array}{l}\text { Standard: } \\
\text { Sample type: }\end{array}$ & $\begin{array}{c}\text { AGV-1 } \\
\text { Andesite }\end{array}$ & $\begin{array}{c}\text { All92-29-1 } \\
\text { Basalt }\end{array}$ & $\begin{array}{l}\text { Bas-III504B } \\
\text { Basalt }\end{array}$ & $\begin{array}{l}\text { BE-N } \\
\text { Basalt }\end{array}$ & $\begin{array}{c}\text { BHVO-1 } \\
\text { Basalt }\end{array}$ & $\begin{array}{l}\text { BIR-1 } \\
\text { Basalt }\end{array}$ & $\begin{array}{c}\text { BR } \\
\text { Basalt }\end{array}$ & $\begin{array}{l}\text { DR-N } \\
\text { Diorite }\end{array}$ & $\begin{array}{c}\text { G-2 } \\
\text { Granite }\end{array}$ & $\begin{array}{l}\text { GBM-1 } \\
\text { Gamet }\end{array}$ & $\begin{array}{c}\text { GH } \\
\text { Granite }\end{array}$ & $\begin{array}{c}\text { MAG-1 } \\
\text { Marine Mud }\end{array}$ & $\begin{array}{l}\text { MRG-1 } \\
\text { Gabbro }\end{array}$ & $\begin{array}{c}\text { NBS-688 } \\
\text { Basalt }\end{array}$ & $\begin{array}{l}\text { NIM-D } \\
\text { Dunite }\end{array}$ & $\begin{array}{c}\text { PCC-1 } \\
\text { Peridotite }\end{array}$ & $\begin{array}{l}\text { PG-721 } \\
\text { Feldspar }\end{array}$ & $\begin{array}{l}\text { RGM-1 } \\
\text { Rhyolite }\end{array}$ & $\begin{array}{l}\text { STM-1 } \\
\text { Syenite }\end{array}$ & $\begin{array}{l}\text { UB-N } \\
\text { Serpent. }\end{array}$ \\
\hline $\begin{array}{l}\mathrm{Nb} \mathrm{RV} \\
\mathrm{Nb} F \mathrm{~F} \\
\mathrm{NbSD}\end{array}$ & $\begin{array}{l}15 \\
13.9 \\
0.40\end{array}$ & & $\begin{array}{l}0.60 \\
0.46 \\
0.14\end{array}$ & $\begin{array}{l}100 \\
112.4 \\
1.3\end{array}$ & $\begin{array}{l}19 \\
18.7 \\
0.25\end{array}$ & $\begin{array}{l}2.0 \\
0.37 \\
0.19\end{array}$ & $\begin{array}{c}98 \\
110 \\
1.1\end{array}$ & $\begin{array}{l}8.0 \\
7.4 \\
0.26\end{array}$ & $\begin{array}{l}12 \\
12 \\
0.46\end{array}$ & $\begin{array}{l}1.5 \\
1.4 \\
0.16\end{array}$ & $\begin{array}{l}85 \\
92 \\
1.3\end{array}$ & $\begin{array}{l}12 \\
15.6 \\
0.44\end{array}$ & $\begin{array}{l}20 \\
22.3 \\
2.3\end{array}$ & & & $\begin{array}{l}1 \\
0.04 \\
0.17\end{array}$ & & $\begin{array}{l}9.2 \\
9.3 \\
0.29\end{array}$ & $\begin{array}{l}271 \\
274 \\
7.8\end{array}$ & \\
\hline $\begin{array}{l}\mathrm{ZrRV} \\
\mathrm{ZrFV} \\
\mathrm{ZrSD}\end{array}$ & $\begin{array}{l}227 \\
238 \\
5.6\end{array}$ & & $\begin{array}{l}40.3 \\
39.4 \\
0.46\end{array}$ & $\begin{array}{l}265 \\
286 \\
3.3\end{array}$ & $\begin{array}{l}179 \\
179 \\
1.9\end{array}$ & $\begin{array}{l}22 \\
15 \\
0.4\end{array}$ & $\begin{array}{l}250 \\
282 \\
2.7\end{array}$ & $\begin{array}{l}125 \\
127 \\
2.2\end{array}$ & $\begin{array}{l}309 \\
323 \\
4.4\end{array}$ & $\begin{array}{l}95 \\
94 \\
0.78\end{array}$ & $\begin{array}{l}150 \\
154 \\
3.9\end{array}$ & $\begin{array}{l}126 \\
131 \\
2.1\end{array}$ & $\begin{array}{l}108 \\
108 \\
1.6\end{array}$ & $\begin{array}{l}60.6 \\
56.5 \\
0.46\end{array}$ & $\begin{array}{l}3.0 \\
3.0 \\
0.52\end{array}$ & $\begin{array}{l}0.3 \\
0.017 \\
0.3\end{array}$ & & $\begin{array}{l}243 \\
241 \\
2.5\end{array}$ & $\begin{array}{r}1345 \\
1361 \\
13\end{array}$ & $\begin{array}{l}8 \\
3.7 \\
0.37\end{array}$ \\
\hline $\begin{array}{l}\text { Y RV } \\
\text { Y FV } \\
\text { Y SD }\end{array}$ & $\begin{array}{l}20 \\
18.2 \\
\quad .99\end{array}$ & & $\begin{array}{c}19.8 \\
20 \\
0.34\end{array}$ & $\begin{array}{l}30 \\
28.5 \\
0.70\end{array}$ & $\begin{array}{c}27.6 \\
26.0 \\
0.44\end{array}$ & $\begin{array}{l}16 \\
15.4 \\
0.52\end{array}$ & $\begin{array}{l}30 \\
28.6 \\
0.60\end{array}$ & $\begin{array}{l}28 \\
25.4 \\
0.66\end{array}$ & $\begin{array}{l}11 \\
9.2 \\
.86\end{array}$ & $\begin{array}{c}30.7 \\
30.9 \\
0.52\end{array}$ & $\begin{array}{l}75 \\
77 \\
2.8\end{array}$ & $\begin{array}{l}28 \\
25.3 \\
1.3\end{array}$ & $\begin{array}{l}14 \\
12.5 \\
0.58\end{array}$ & $\begin{array}{l}17 \\
19.4 \\
0.31\end{array}$ & & $\begin{array}{c}0.1 \\
-0.12 \\
0.37\end{array}$ & & $\begin{array}{l}24 \\
23.1 \\
0.38\end{array}$ & $\begin{array}{l}46 \\
45.3 \\
1.3\end{array}$ & $\begin{array}{l}2.5 \\
2.2 \\
0.31\end{array}$ \\
\hline $\begin{array}{l}\text { Sr RV } \\
\text { SrFV } \\
\text { Sr SDD }\end{array}$ & $\begin{array}{r}662 \\
647 \\
16\end{array}$ & $\begin{array}{c}129 \\
129 \\
1.5\end{array}$ & $\begin{array}{c}60.6 \\
60.4 \\
.89\end{array}$ & $\begin{array}{r}1370 \\
1379 \\
19\end{array}$ & $\begin{array}{l}403 \\
395 \\
3.6\end{array}$ & $\begin{array}{l}108 \\
109 \\
0.6\end{array}$ & $\begin{array}{r}1320 \\
1356 \\
19\end{array}$ & $\begin{array}{l}400 \\
391 \\
4.3\end{array}$ & $\begin{array}{l}478 \\
482 \\
1.6\end{array}$ & $\begin{array}{l}9.7 \\
9.2 \\
.89\end{array}$ & $\begin{array}{l}10 \\
9.0 \\
0.05\end{array}$ & $\begin{array}{c}146 \\
139 \\
3.6\end{array}$ & $\begin{array}{r}266 \\
271 \\
2.8\end{array}$ & $\begin{array}{c}169.2 \\
168 \\
1.7\end{array}$ & $\begin{array}{l}3 \\
3.1 \\
0.90\end{array}$ & $\begin{array}{l}0.4 \\
0.48 \\
0.60\end{array}$ & $\begin{array}{l}809 \\
794 \\
5.1\end{array}$ & $\begin{array}{l}106 \\
104 \\
1.3\end{array}$ & $\begin{array}{r}716 \\
710 \\
12\end{array}$ & $\begin{array}{l}10 \\
7.1 \\
0.4\end{array}$ \\
\hline $\begin{array}{l}\mathrm{Rb} \text { RV } \\
\mathrm{RbFV} \\
\mathrm{RbSD}\end{array}$ & $\begin{array}{c}67.3 \\
66 \\
1.8\end{array}$ & $\begin{array}{l}1.2 \\
0.73 \\
0.82\end{array}$ & $\begin{array}{c}0.1 \\
-0.2 \\
0.52\end{array}$ & $\begin{array}{l}47 \\
46.7 \\
0.22\end{array}$ & $\begin{array}{l}11 \\
8.8 \\
0.61\end{array}$ & $\begin{array}{l}0.40 \\
0.083 \\
0.80\end{array}$ & $\begin{array}{l}47 \\
47 \\
0.83\end{array}$ & $\begin{array}{l}73 \\
71 \\
0.32\end{array}$ & $\begin{array}{l}170 \\
169 \\
0.54\end{array}$ & $\begin{array}{l}1.2 \\
0.75 \\
0.89\end{array}$ & $\begin{array}{c}390 \\
383 \\
1.7\end{array}$ & $\begin{array}{l}149 \\
148 \\
0.85\end{array}$ & $\begin{array}{l}8.5 \\
7.0 \\
0.45\end{array}$ & $\begin{array}{l}1.91 \\
1.67 \\
0.56\end{array}$ & & $\begin{array}{c}0.066 \\
-0.05 \\
0.72\end{array}$ & $\begin{array}{l}0.90 \\
0.78 \\
0.24\end{array}$ & $\begin{array}{l}150 \\
150 \\
0.92\end{array}$ & $\begin{array}{l}118 \\
117 \\
2.6\end{array}$ & $\begin{array}{l}6 \\
3.3 \\
0.55\end{array}$ \\
\hline $\begin{array}{l}\mathrm{Zn} R \mathrm{RV} \\
\mathrm{ZnFV} \\
\mathrm{ZnSD}\end{array}$ & $\begin{array}{l}88 \\
93 \\
5.5\end{array}$ & & $\begin{array}{l}69 \\
63 \\
4.6\end{array}$ & $\begin{array}{l}120 \\
124 \\
2.7\end{array}$ & $\begin{array}{l}105 \\
108 \\
2.2\end{array}$ & $\begin{array}{l}71 \\
70 \\
2.1\end{array}$ & $\begin{array}{l}160 \\
161 \\
3.7\end{array}$ & $\begin{array}{r}145 \\
162 \\
13\end{array}$ & $\begin{array}{l}86 \\
87 \\
1.9\end{array}$ & $\begin{array}{c}75 \\
65 \\
6.4\end{array}$ & $\begin{array}{l}85 \\
57 \\
2.1\end{array}$ & $\begin{array}{l}130 \\
139 \\
2.2\end{array}$ & $\begin{array}{l}191 \\
213 \\
2.2\end{array}$ & $\begin{array}{c}58 \\
73 \\
4.3\end{array}$ & $\begin{array}{l}90 \\
94 \\
3.2\end{array}$ & $\begin{array}{l}42 \\
43 \\
2.2\end{array}$ & & $\begin{array}{l}32 \\
32 \\
2.1\end{array}$ & $\begin{array}{l}244 \\
250 \\
7.5\end{array}$ & $\begin{array}{l}85 \\
86 \\
1.6\end{array}$ \\
\hline $\begin{array}{l}\text { Cu RV } \\
\text { Cu FV } \\
\text { Cu SD }\end{array}$ & $\begin{array}{l}60 \\
60 \\
3.3\end{array}$ & $\begin{array}{l}64 \\
65 \\
1.6\end{array}$ & $\begin{array}{l}91 \\
89 \\
3.3\end{array}$ & $\begin{array}{l}72 \\
73 \\
3.3\end{array}$ & $\begin{array}{l}136 \\
137 \\
2.0\end{array}$ & $\begin{array}{l}126 \\
129 \\
2.5\end{array}$ & $\begin{array}{l}72 \\
73 \\
2.7\end{array}$ & $\begin{array}{l}50 \\
49 \\
0.97\end{array}$ & $\begin{array}{l}11 \\
12 \\
2.8\end{array}$ & & $\begin{array}{l}14 \\
3.0 \\
2.3\end{array}$ & $\begin{array}{l}30 \\
31 \\
1.4\end{array}$ & $\begin{array}{l}134 \\
131 \\
3.4\end{array}$ & $\begin{array}{l}96 \\
94 \\
2.4\end{array}$ & $\begin{array}{l}10 \\
13 \\
1.6\end{array}$ & $\begin{array}{l}10 \\
8 \\
0.87\end{array}$ & & $\begin{array}{l}12 \\
12 \\
1.2\end{array}$ & $\begin{array}{l}5 \\
1.8 \\
6.1\end{array}$ & $\begin{array}{l}28 \\
22 \\
0.98\end{array}$ \\
\hline $\begin{array}{l}\mathrm{Ni} \text { RV } \\
\mathrm{Ni} \text { FV } \\
\mathrm{Ni} \text { SD }\end{array}$ & $\begin{array}{l}16 \\
15.6 \\
0.84\end{array}$ & & $\begin{array}{l}128 \\
129 \\
0.97\end{array}$ & $\begin{array}{l}267 \\
280 \\
1.4\end{array}$ & $\begin{array}{l}121 \\
120 \\
1.8\end{array}$ & $\begin{array}{l}166 \\
160 \\
1.0\end{array}$ & $\begin{array}{l}260 \\
271 \\
2.2\end{array}$ & $\begin{array}{l}15 \\
16 \\
1.2\end{array}$ & $\begin{array}{l}5.5 \\
5.0 \\
1.1\end{array}$ & & $\begin{array}{l}3 \\
7.6 \\
6.4\end{array}$ & $\begin{array}{l}53 \\
55 \\
2.1\end{array}$ & $\begin{array}{l}193 \\
193 \\
1.8\end{array}$ & $\begin{array}{l}150 \\
140 \\
2.1\end{array}$ & $\begin{array}{r}2050 \\
2094 \\
27\end{array}$ & $\begin{array}{r}2380 \\
2471 \\
19\end{array}$ & & $\begin{array}{l}4 \\
9.5 \\
3.6\end{array}$ & $\begin{array}{l}3 \\
4.9 \\
1.6\end{array}$ & $\begin{array}{r}2000 \\
2009 \\
18\end{array}$ \\
\hline $\begin{array}{l}\mathrm{CrRV} \\
\mathrm{CrFV} \\
\mathrm{Cr} \text { SD }\end{array}$ & $\begin{array}{r}10.1 \\
6.2 \\
2.3\end{array}$ & & $\begin{array}{l}374 \\
369 \\
3.6\end{array}$ & $\begin{array}{l}360 \\
374 \\
1.1\end{array}$ & $\begin{array}{l}289 \\
290 \\
2.0\end{array}$ & $\begin{array}{l}382 \\
377 \\
6.3\end{array}$ & $\begin{array}{l}380 \\
342 \\
5.6\end{array}$ & $\begin{array}{l}42 \\
32 \\
4.0\end{array}$ & $\begin{array}{l}8.7 \\
4.1 \\
3.5\end{array}$ & $\begin{array}{l}23 \\
24 \\
5.2\end{array}$ & $\begin{array}{c}6 \\
-3.0 \\
3.7\end{array}$ & $\begin{array}{c}97 \\
101 \\
2.5\end{array}$ & $\begin{array}{l}526 \\
528 \\
1.5\end{array}$ & $\begin{array}{l}332 \\
293 \\
5.8\end{array}$ & $\begin{array}{r}2900 \\
2880 \\
16\end{array}$ & $\begin{array}{r}2730 \\
2490 \\
38\end{array}$ & & $\begin{array}{r}3 \\
15 \\
10\end{array}$ & $\begin{array}{c}4 \\
-1.4 \\
4.2\end{array}$ & $\begin{array}{r}2300 \\
2400 \\
27\end{array}$ \\
\hline $\begin{array}{l}\mathrm{TiO}_{2} \mathrm{RV} \\
\mathrm{TiO}_{2} \mathrm{FV} \\
\mathrm{TiO}_{2} \mathrm{SD}\end{array}$ & $\begin{array}{l}1.05 \\
1.03 \\
0.023\end{array}$ & $\begin{array}{l}1.77 \\
1.80 \\
0.030\end{array}$ & & $\begin{array}{l}2.6 \\
2.8 \\
.066\end{array}$ & $\begin{array}{l}2.71 \\
2.79 \\
0.065\end{array}$ & $\begin{array}{l}0.96 \\
0.95 \\
0.009\end{array}$ & $\begin{array}{l}2.6 \\
2.7 \\
0.060\end{array}$ & $\begin{array}{l}1.09 \\
1.1 \\
0.008\end{array}$ & $\begin{array}{l}0.48 \\
0.46 \\
0.010\end{array}$ & $\begin{array}{l}0.23 \\
0.24 \\
0.001\end{array}$ & $\begin{array}{l}0.08 \\
0.078 \\
0.003\end{array}$ & $\begin{array}{l}0.75 \\
0.73 \\
0.008\end{array}$ & $\begin{array}{l}3.77 \\
4.07 \\
0.13\end{array}$ & $\begin{array}{l}1.17 \\
1.13 \\
0.013\end{array}$ & $\begin{array}{l}0.02 \\
0.02 \\
0.001\end{array}$ & $\begin{array}{l}0.004 \\
0.001 \\
0.002\end{array}$ & & $\begin{array}{l}0.27 \\
0.27 \\
0.003\end{array}$ & & $\begin{array}{l}0.11 \\
0.096 \\
0.004\end{array}$ \\
\hline $\begin{array}{l}\text { Ce RV } \\
\text { Ce FV } \\
\text { Ce SD }\end{array}$ & $\begin{array}{l}67 \\
63 \\
4.1\end{array}$ & $\begin{array}{l}12 \\
15 \\
2.0\end{array}$ & & $\begin{array}{l}152 \\
158 \\
2.8\end{array}$ & $\begin{array}{l}38 \\
42 \\
1.8\end{array}$ & $\begin{array}{l}2.5 \\
1.1 \\
5.1\end{array}$ & $\begin{array}{c}151 \\
151 \\
3.8\end{array}$ & $\begin{array}{l}46 \\
49 \\
13\end{array}$ & $\begin{array}{c}160 \\
161 \\
2.9\end{array}$ & & $\begin{array}{l}60 \\
47 \\
0.52\end{array}$ & $\begin{array}{l}88 \\
74 \\
13\end{array}$ & $\begin{array}{l}26 \\
16 \\
7.2\end{array}$ & $\begin{array}{c}13 \\
9.5 \\
3.4\end{array}$ & & $\begin{array}{l}0.1 \\
0.95 \\
3.4\end{array}$ & & $\begin{array}{l}47 \\
45 \\
14\end{array}$ & $\begin{array}{r}259 \\
248 \\
10\end{array}$ & $\begin{array}{r}1.0 \\
-1.6 \\
6.2\end{array}$ \\
\hline $\begin{array}{l}\text { Ba RV } \\
\text { BaFV } \\
\text { BaSD }\end{array}$ & $\begin{array}{r}1226 \\
1201 \\
41\end{array}$ & & $\begin{array}{l}0.8 \\
2.8 \\
2.4\end{array}$ & $\begin{array}{r}1025 \\
1104 \\
19\end{array}$ & $\begin{array}{r}128 \\
8\end{array}$ & $\begin{array}{c}6.8 \\
9.2 \\
15\end{array}$ & $\begin{array}{r}1050 \\
1158 \\
23\end{array}$ & $\begin{array}{r}373 \\
13\end{array}$ & $\begin{array}{r}1861 \\
35\end{array}$ & $\begin{array}{r}10 \\
0 \\
5\end{array}$ & $\begin{array}{c}13 \\
4.6\end{array}$ & $\begin{array}{r}463 \\
10\end{array}$ & $\begin{array}{l}49 \\
5.7\end{array}$ & $\begin{array}{r}183 \\
4.8\end{array}$ & $\begin{array}{l}10 \\
-3.0 \\
7.0\end{array}$ & $\begin{array}{l}1.2 \\
1.1 \\
3.9\end{array}$ & & $\begin{array}{r}807 \\
808 \\
25\end{array}$ & $\begin{array}{r}570 \\
587 \\
19\end{array}$ & $\begin{array}{l}30 \\
21 \\
14\end{array}$ \\
\hline $\begin{array}{l}\text { VRV } \\
\text { VFV } \\
\text { V SD }\end{array}$ & $\begin{array}{c}121 \\
116 \\
4.7\end{array}$ & $\begin{array}{l}293 \\
297 \\
4.9\end{array}$ & $\begin{array}{l}250 \\
247 \\
4.1\end{array}$ & $\begin{array}{l}235 \\
245 \\
8.4\end{array}$ & $\begin{array}{r}317 \\
311 \\
12\end{array}$ & $\begin{array}{l}308 \\
7.8\end{array}$ & $\begin{array}{r}235 \\
279 \\
63\end{array}$ & $\begin{array}{l}220 \\
218 \\
2.4\end{array}$ & $\begin{array}{l}36 \\
39 \\
3.1\end{array}$ & & $\begin{array}{l}5 \\
0.20 \\
0.35\end{array}$ & $\begin{array}{l}140 \\
145 \\
4.8\end{array}$ & $\begin{array}{r}570 \\
21\end{array}$ & $\begin{array}{l}250 \\
228 \\
2.5\end{array}$ & $\begin{array}{l}40 \\
44 \\
3.5\end{array}$ & $\begin{array}{l}31 \\
33 \\
4.0\end{array}$ & & $\begin{array}{r}12 \\
11 \\
3\end{array}$ & $\begin{array}{l}6 \\
1.1 \\
2.2\end{array}$ & $\begin{array}{l}75 \\
64 \\
1.3\end{array}$ \\
\hline
\end{tabular}

Notes: $\mathrm{RV}=$ recommended values; $\mathrm{FV}=$ found values; $\mathrm{SD}=$ standard deviation $(=1 \sigma)$. All concentrations in ppm, except $\mathrm{TiO}_{2}$ (given in wt\%). 
Table 2. Errors in trace element data by XRF analysis.

\begin{tabular}{|c|c|c|c|c|c|}
\hline \multirow[b]{2}{*}{ Element } & \multicolumn{2}{|c|}{ Total number studied: } & \multicolumn{2}{|c|}{$\begin{array}{c}\text { Element concentrations at } \\
\text { which rel. SD equals } \\
\end{array}$} & \multirow{2}{*}{$\begin{array}{c}\text { Lower end of } \\
\text { calibration } \\
\text { curve (Bach, } \\
\text { 1991) }\end{array}$} \\
\hline & $\begin{array}{l}\text { Geological } \\
\text { standards }\end{array}$ & $\begin{array}{c}\text { Element } \\
\text { determinations }\end{array}$ & $30 \%$ & $10 \%$ & \\
\hline $\mathrm{Nb}$ & 15 & 84 & 0.6 & 4 & 5 \\
\hline $\mathrm{Zr}$ & 18 & 100 & 11 & 20 & 10 \\
\hline $\mathrm{Y}$ & 17 & 96 & 1.5 & 5 & 5 \\
\hline $\mathrm{Sr}$ & 20 & 110 & 2 & 8 & 10 \\
\hline $\mathrm{Rb}$ & 19 & 104 & 3 & 10 & 5 \\
\hline $\mathrm{Zn}$ & 18 & 162 & $?$ & 20 & 10 \\
\hline $\mathrm{Cu}$ & 18 & 156 & 8 & 25 & 10 \\
\hline $\mathrm{Ni}$ & 17 & 150 & 3 & 15 & 5 \\
\hline $\mathrm{Cr}$ & 18 & 132 & 8 & 40 & 10 \\
\hline $\mathrm{TiO}_{2}$ & 18 & 128 & $=100$ & 400 & - \\
\hline $\mathrm{Ce}$ & 16 & 112 & 10 & 40 & 一 \\
\hline $\mathrm{Ba}$ & 19 & 136 & 18 & 100 & $=50$ \\
\hline V & 18 & 110 & 16 & 55 & - \\
\hline
\end{tabular}

Notes: All concentrations in ppm. ? = Lack of low-level data makes it hard to estimate the relative errors. $\mathrm{TiO}_{2}$ values were determined together with the trace elements.

This impression is corroborated if we study the accuracy of the $\mathrm{Cu}$ data. Accurate values in an absolute sense are impossible to define for any constituent. However, many users of data from the literature are implicitly assuming that the quoted values are sufficiently good (that is, accurate); otherwise the quotation would not occur. In lack of better estimators we will here define the accuracy $\mathrm{A}$ as the relation:

$$
\mathrm{A}=100 \cdot(\mathrm{RV}-\mathrm{FV}) / \mathrm{RV} \text {; }
$$
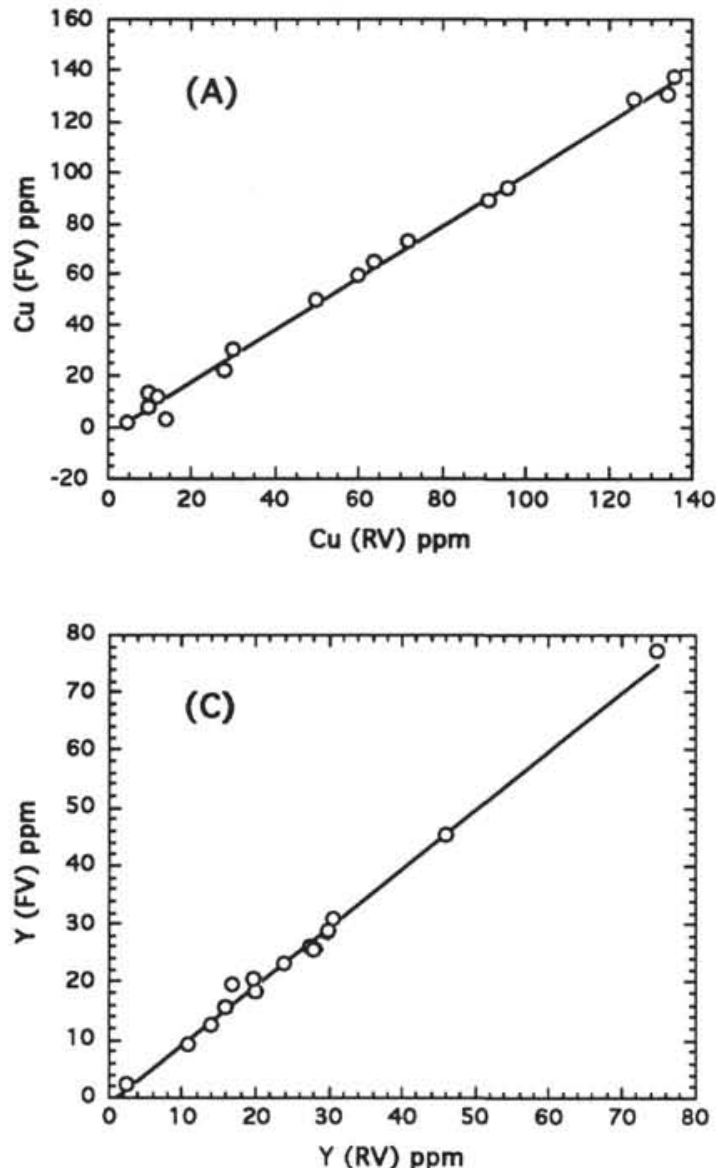

it is obvious that all estimates of accuracies are critically dependent on reference materials of good quality.

Using this definition we find the accuracy of the $\mathrm{Cu}$ data given in Figure $3 \mathrm{~A}$ at low concentrations, which gives an approximate estimate of the accuracy. In this graph the values for $\mathrm{Cu}$ show a sharp drop in quality below about $20-\mathrm{ppm} \mathrm{Cu}$. Thus, values that should read $14 \mathrm{ppm}$ are instead found to be about $3 \mathrm{ppm}$.

The corresponding plots for $\mathrm{Ba}$ are shown in Figures 1B, 2B, and $3 \mathrm{~B}$. The relative errors for $\mathrm{Ba}$ are conspicuous already at the $100-\mathrm{ppm}$ level, and at about $18 \mathrm{ppm}$ they amount to $30 \%$. This suggests that $\mathrm{XRF}$ data for $\mathrm{Ba}$ are generally fairly unreliable below $18 \mathrm{ppm}$. Thus, negative measured values for $\mathrm{Ba}$ correspond to RV data of $10 \mathrm{ppm}$, and the discrepancy RV-FV is about $-8 \mathrm{ppm}$ at the 25 -ppm level.

Data for $\mathrm{Y}$ and $\mathrm{Sr}$, on the other hand, show much better precisions and accuracies, as is demonstrated in Figures $1 \mathrm{C}$ and $1 \mathrm{D}, 2 \mathrm{C}$ and 2D, and $3 \mathrm{C}$ and $3 \mathrm{D}$. Both elements can be determined with a $10 \%$ precision at $5-8 \mathrm{ppm}$ and with a $30 \%$ precision at 1.5 to $2.0 \mathrm{ppm}$. Although there is no direct connection between precision and accuracy, there is nevertheless a pronounced correlation between these determinations, with poor precisions generally being associated with poor accuracies.

Using similar graphs for all other data in Table 1 one may also study the FV, SD, and RV relations for the remaining elements and their "accuracies." In some cases the interpretation of these other diagrams is fairly simple (e.g., for $\mathrm{Nb}$ ) whereas in other cases the error distributions are poorly defined (Ce, for instance). Most RV-FV plots show only little scatter $(1 \%-3 \%)$ about the regression line at high recommended values, but $\mathrm{Zn}$ may sporadically show deviations of up to $5 \%$ even at fairly high concentrations. Possibly this is caused by disturbances due to variations in pressure (Bertin, 1975).
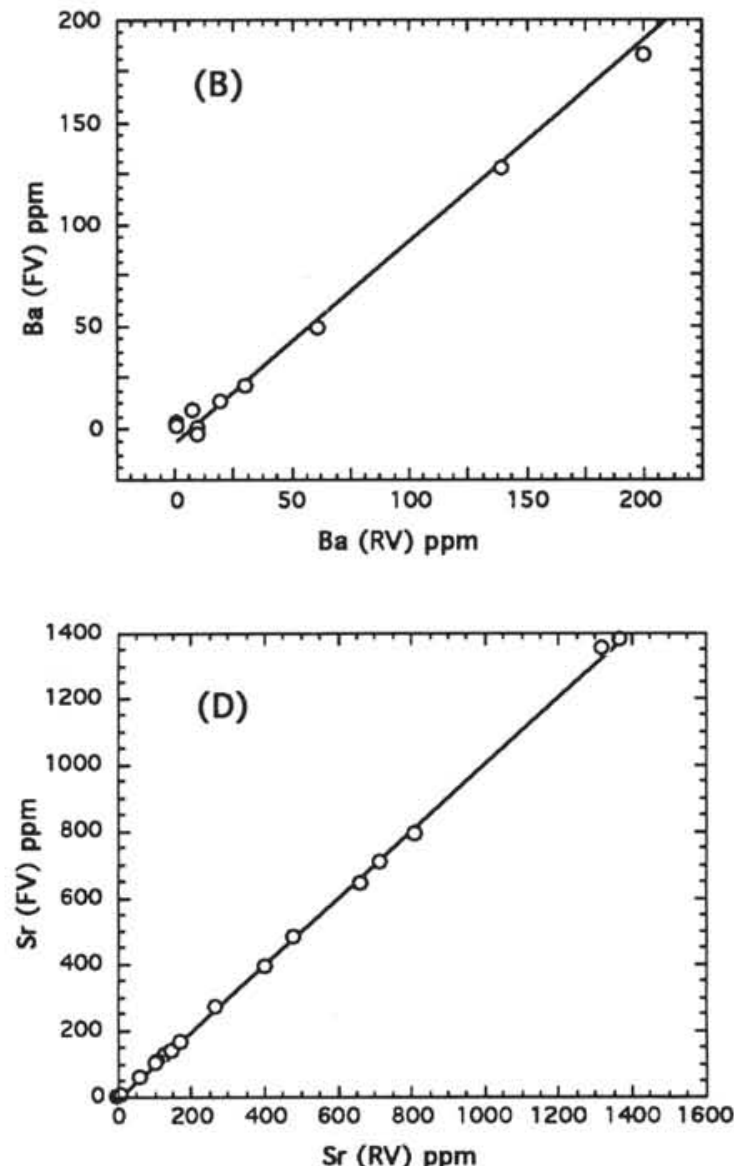

Figure 1. Recommended values (RV) and found mean values $(\mathrm{FV})$ for $\mathrm{Cu}, \mathrm{Ba}, \mathrm{Y}$, and $\mathrm{Sr}$ determined by XRF and based on data in Table 1. Note the scatter in the FV values at low RV levels for $\mathrm{Cu}$ and $\mathrm{Ba}$. The regression lines (over the total concentration range in Table 1 ) have the correlation coefficients $0.998(\mathrm{Cu}), 0.998$ (Ba), $0.997(\mathrm{Y})$, and $1.00(\mathrm{Sr})$. 

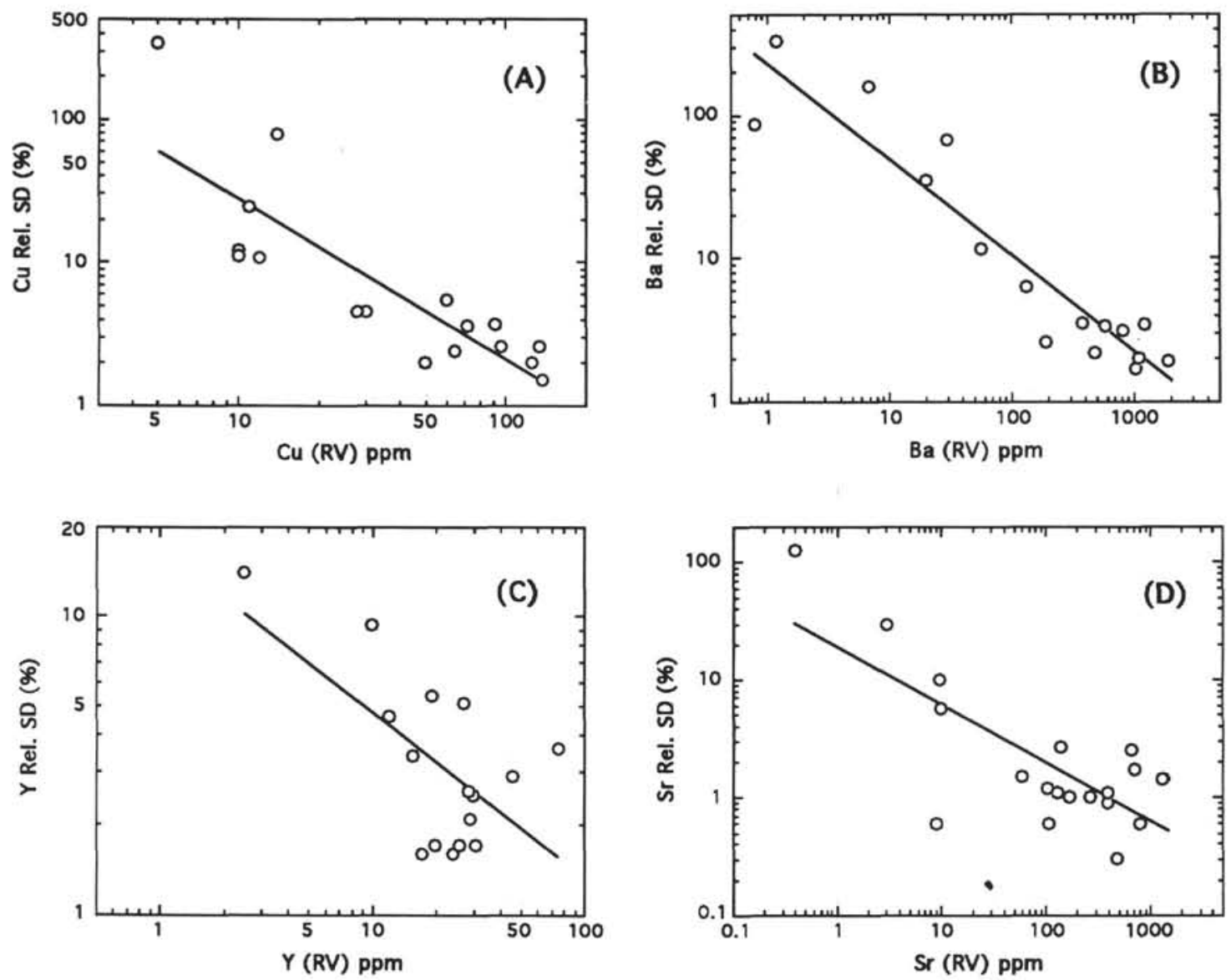

Figure 2. Standard deviations (SD) of the mean determinations by XRF. Rel. SD represents 1 standard deviation given as the relative error in \% of RV. For further details, see text. The regression lines have the correlation coefficients $-0.84(\mathrm{Cu}),-0.94(\mathrm{Ba}),-0.78(\mathrm{Y})$, and -0.96 ( $\mathrm{Sr}$ ). Note that the presence of a correlation coefficient near 1.0 , as for $\mathrm{Ba}$, does not guarantee an excellent detection limit.

The results of these studies are summarized in Table 2, which shows at what concentrations we could expect relative errors (precisions) of $30 \%$ and $10 \%$. In the same table we show the low-end values for calibration curves used for XRF analyses (Bach, 1991); these are not detection limits sensu stricto, but show a general correlation with our more precisely defined relative error. In summary it appears that the elements $\mathrm{Nb}, \mathrm{Y}, \mathrm{Sr}, \mathrm{Rb}$, and $\mathrm{Ni}$ are well suited for determination by $\mathrm{XRF}$, whereas $\mathrm{Cr}, \mathrm{Ce}, \mathrm{Ba}$, and $\mathrm{V}$ yield poorer data, and $\mathrm{Ni}$, $\mathrm{Cu}, \mathrm{Zn}$, and $\mathrm{Zr}$ form a group of intermediary analytical suitability.

It could be argued that some data in Figures 1-3 show too large scatter to support the statistical analyses we perform here. However, it is hard to expect better correlations in many cases, because errors by their very nature show an erratic behavior. A survey of all log-log plots of SD vs. RV reveals that at high RV values, SD tends to show a small error that varies little over a major concentration interval. As the concentrations drop, the SD values steadily increase and scatter about a straight line with a negative slope, which indicates large errors at low RV values. This observation has been made by many analysts, including Massart et al. (1988, figure 3). Thus, at low concentrations, the background variations take on an increasing significance because element peaks are harder to separate from the background. When the peak is smaller than 2-3 times the SD of the background noise, most analytical chemists consider the signal not significant (Skoog and West, 1982; Thompson and Walsh, 1983; Massart et al., 1988).

These results are seemingly at variance with the behavior of some low FV values, which show an apparent good agreement with RV values. However, all FV data in Table 1 represent mean data based on several determinations, usually 6-8. During most routine analyses, on the other hand, only one or two determinations are made of each component, which increases the risk for poor data. Indeed, mean values without the additional information about their SD values may yield completely erroneous impressions (Massart et al., 1988). However, the scatter does not derive exclusively from shipboard data; some of it may be caused by poor recommended values.

\section{DISCUSSION AND CONCLUSIONS}

\section{The Potential of XRF Methods}

Personal contacts and discussions with individuals at various analytical laboratories (e.g., the Geological Survey of Sweden and the Smithsonian Institution, Department of Meteorites) corroborate the results in Table 2. However, these results are not unique for the XRF data obtained aboard the JOIDES Resolution. Using Ba data by Sims et al. (1988) and Verma et al. (1992) we find a similar FV-SD pattern as in Figure 2B; these two data-sets yield a plot for Ba that is almost identical with that in Figure 2B. Plots of their data for $\mathrm{Zr}$ and $\mathrm{Cr}$ yield the same negative slope pattern we found, but the corresponding curves are displaced, bracketing our curves for these elements. Thus, the data by Verma et al. (1992) suggest better reproducibilities than is suggested by the JOIDES Resolution data, whereas results by Sims et al. (1988) suggest poorer reproducibilities. 

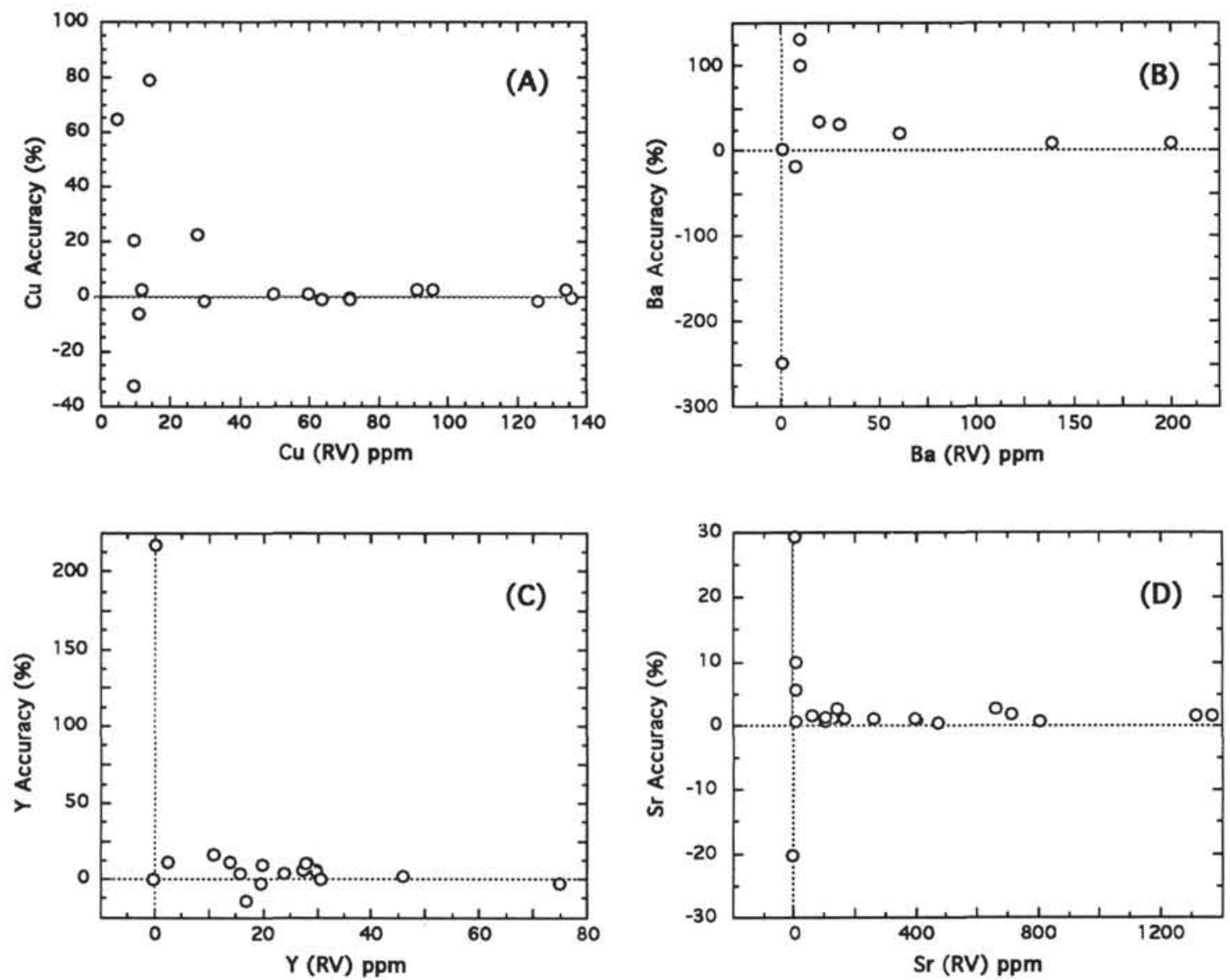

Figure 3. Accuracies of XRF determinations for $\mathrm{Cu}, \mathrm{Ba}, \mathrm{Y}$, and $\mathrm{Sr}$. Accuracy is defined as $100 \cdot(\mathrm{RV}-\mathrm{FV}) / \mathrm{RV}$.

A simplistic interpretation would be that the data by Sims et al. (1988) should be rejected because it is older, but recent reports are not always better than older results. Furthermore, Sims, Gladney, et al. represent a research team well known for many reliable reports. Also, the SD-FV curves based on data in Sims et al. (1988) are very consistent (e.g., as in Figures 2A and 2B), whereas the plot for $\mathrm{Zr}$ data in Verma et al. (1992) shows a worse scatter than that in Figure 2C. These criteria could indicate that the data by Sims et al. (1988) are of better quality, but a final conclusion is hard to reach at present without further study of the analytical routines at the various laboratories. However, the quality of the data from the ship's XRF facility falls between the extremes discussed above, suggesting that this XRF unit is producing the same high-quality data as found in many shore-based XRF laboratories; this was concluded also by Hergt and Sims (1994).

These results are furthermore supported by the findings of Normand et al. (1989) and Boström and Bach (this volume, table 5) that show that most major and several minor component determinations by XRF correspond well to data obtained by atomic emission spectrometry with inductively coupled plasma as excitation source (ICPAES) or instrumental neutron activation analysis (INAA). However, the quality of the analytical data for some trace elements in the Initial Reports has been overestimated during some ODP cruises (Shipboard Scientific Party, 1992c, 1992d, 1992b). This conclusion is further supported by data in Govindaraju (1989a; e.g., for the standards UB-N and BX-N), showing that different methods yield considerably different results even in the 30 to $80 \mathrm{ppm}$ range for $\mathrm{Ba}$. This instrument-related problem is frequently observed but often poorly understood (Hunt and Wilson, 1986; Abbey, 1991).

\section{Alternative Analytical Methods}

The performance of the XRF technique probably cannot be improved much for many trace elements without internal standards or substantial pre-enrichment procedures in the laboratory. Similar observations regarding the lack of low-level determinations by XRF have been made in several geological surveys and research organizations (K. Govindaraju, C. Pontér, C. Papavassiliou, and A. Horowitz, pers. comm., 1975-1993) and explain the accelerating tendency, particularly since around 1980, to introduce ICP-AES methods (Burman et al., 1977; Walsh, 1980; Montaser, 1992). Furthermore, many very sensitive mass spectrometers using ICP sources exist now (Horlick and Shao, 1992). However, they are still expensive and require special clean laboratories for optimal use.

It can be argued that XRF instruments like the present ARL unit on the ship will provide good service in the near future as long as there is no pressing need to measure several trace elements such as $\mathrm{Ba}, \mathrm{Cu}, \mathrm{La}, \mathrm{Y}$, and $\mathrm{Yb}$ with precision down to the $0.5-5 \mathrm{ppm}$ level. We agree with this conclusion, but we also feel that XRF methods have too poor sensitivity for some trace components and lack sufficient versatility for many of the interesting and expanded geochemical programs that could be run on the JOIDES Resolution.

ICP-AES and atomic absorption spectrometry (AA) instruments are in many ways superior to XRF, but require laboratory personnel of greater skill than is needed for XRF. This is particularly the case with AA methods because of the numerous complications resulting from the matrix problems. The fact that AA units are used on the JOIDES Resolution suggests that an ICP-AES unit might also operate 
well aboard ship. Furthermore, one of us (K.B.) has noted that AA units may be more sensitive to vibrations than emission spectrometers. Indeed, these ICP-AES systems may be remarkably rugged; K.B. was present when a unit fell during unloading, yet operated flawlessly a few hours later! Furthermore, the use of nonflammable gases would further increase safety in the laboratory.

As to the choice between AA and ICP-AES, a growing number of geochemists are using ICP-AES methods. Superficially ICP-AES methods have poorer detection limits than, for instance, AA units with graphite furnace attachment (Welz, 1985), but this is commonly only the case when the samples consist of very dilute solutions; the analysis of most real samples by AA is much slower and more disturbed by matrix effects than by ICP-AES. The latter method can provide a complete rock analysis involving about 30-40 element determinations in about 1-2 minutes in a multichannel instrument, compared with a 10-times-longer period in an AA unit and 100 times longer by XRF. Furthermore, ICP-AES systems are much less demanding concerning sample sizes and can routinely analyze samples as small as $100-250 \mathrm{mg}$; under special circumstances these limits can be pushed down to a few milligrams for a selected number (10-15) of elements of interest (for instance, in separate minerals and phenocrysts). These aspects have been further discussed in Burman et al. (1977, 1978), in Burman and Boström (1979), Thompson and Walsh (1983), and Boström, Perissoratis et al. (1990). ICP-AES can, in addition, perform chemical analyses of metal-enriched seawaters and hydrothermal plumes (Boström, Ingri, et al., 1990) and several rare earth elements (Boström, 1987).

Furthermore, ongoing problems regarding instrumental drift and precision in the data can, to a large extent, be reduced by means of internal standards, a procedure that has been used for decades in emission spectrometry (Ahrens and Taylor, 1961); in the ICP-AES techniques it implies that the precisions of major elements may routinely be measured with a relative SD of about $0.2 \%-0.4 \%$ for more elements than is the case for XRF and at lower levels (C. Pontér, pers. comm., 1993; K. Boström, unpubl. data). Furthermore, modern approaches to correct for drift (Mermet, 1992) make drift problems much less of a nuisance than earlier.

\section{RECOMMENDATIONS AND COMMENTS}

Statistical tests of obtained geochemical results, including XRF data, should be performed more widely aboard JOIDES Resolution. The uncertainties in the calibration curves should be explicitly expressed together with all final results as pointed out above and in Flanagan (1986) (e.g., as Sy.x.), following routines that are used in many onshore chemical laboratories. Most optimistic claims of precision and accuracy can be avoided if the analytical errors are considered.

Similar opinions have been expressed by Dick, Erzinger, Stokking, et al., (1992). Their study of the data for ODP/DSDP Hole 504B indicates that the interlaboratory variability of samples is surprisingly high, even when only XRF analyses are considered. For instance, $\mathrm{Ti} / \mathrm{Zr}$ ratios seem to differ considerably with depth in the lower part of the hole. This variability, in fact, is merely the result of systematic differences in the determination of $\mathrm{Ti}$ and $\mathrm{Zr}$ in various laboratories. Though all data are claimed to be precise, the accuracies are rarely reported in most geoscience papers, and this makes the combination of data difficult. The compilation of data demands particularly good precision and accuracy for the studies of ODP/DSDP holes that are visited repeatedly over a considerable span of time and are studied by different laboratories. This problem can be overcome only if all analysts consistently report their data for widely used reference rocks (e.g., BHVOI-1 and BCR-1); this procedure should therefore be routine for all ODP reports. This was done during Leg 142 (Storms, Batiza, et al. 1993) but not during many other ODP legs.

\section{ACKNOWLEDGMENTS}

We want to thank Mr. Kazushi (Kuro) Kuroki, for cordial assistance in locating the data needed for this study, which was started aboard the ship. His exemplary work and order in the XRF facility on board was a prerequisite for the successful study of these analytical problems. We furthermore want to thank Dr J. Allan of ODP for kindly supplying us with an advance copy of the paper by Hergt and Sims (1994), which was unknown to us when we started this paper on the JOIDES Resolution.

This research was supported by a grant to K.B. from the Swedish Natural Sciences Research Council. This support is gratefully acknowledged.

\section{REFERENCES}

Abbey, S., 1988. Robust measures and the estimator limit. Geostand. Newsl., $12: 241-248$.

, 1991. What is a "Method"? Geostand. Newsl., 15:191-194.

Ahrens, L.H., and Taylor, S.R., 1961. Spectrochemical Analysis (2nd ed.): Reading, MA (Addison-Wesley).

Arvanitides, N., Boström, K., Kalogeropoulos, S., Paritsis, S., Galanopoulos, V.P., and Papavassiliou, C., 1990. Geochemistry of lavas, pumice and veins in drillcore GPK-1, Palaea Kameni, Santorini. In Hardy, D.A., Keller, J., Galanopoulos, V.P., Flemming, N.C., Druitt, T.H. (Eds.), Thera and the Aegean World, III (Vol. 2): London (The Thera Foundation), 266-279.

Bach, W., 1991. Geochemie und Petrogenese von Ozeanbodenbasalten des Ostpazifischen Rückens $6^{\circ}-30^{\circ} \mathrm{S}$ [Diploma (M.S.) thesis]. Justus-Liebig Univ., Giessen.

Bertin, E.P., 1975. Principles and Practice of X-ray Spectrometric Analysis (2nd ed.): New York (Plenum).

Boström, B., 1987. Report on Ailsa Craig Granite. In Govindaraju, K. (Ed.), 1987 Compilation Report on Ailsa Craig Granite AC-E with the Participation of 128 GIT-IWG Laboratories. Geostand. Newsl., 11:203-255.

Boström, K., Ingri, J., Boström, B., Andersson, P., and Löfvendahl, R., 1990. Metallogenesis at Santorini: a subduction-zone related process. II: Geochemistry and origin of hydrothermal solutions on Nea Kameni, Santorini, Greece. In Hardy, D.A., Keller, J., Galanopoulos, V.P., Flemming, N.C., Druitt, T.H. (Eds.), Thera and the Aegean World, III (Vol. 2): London (The Thera Foundation), 291-299.

Boström, K., Perissoratis, C., Galanopoulos, V.P., Papavassiliou, C., Boström, B., Ingri, J., and Kalogeropoulos, S.,1990. Geochemistry and structural control of hydrothermal sediments and new hot springs in the Caldera of Santorini, Greece. In Hardy, D.A., Keller, J., Galanopoulos, V.P., Flemming, N.C., Druitt, T.H. (Eds.), Thera and the Aegean World, III (Vol. 3): London (The Thera Foundation), 325-336.

Burman, J.O., Boström, B., and Boström, K., 1977. Geochemical analysis by plasma spectroscopy. Geol. Foren. Stockh. Forhandl., 99:102-110.

Burman, J.O., and Boström, K., 1979. Comparison of different plasma excitation and calibration methods in the analyses of geological materials by optical emission spectrometry. Anal. Chem., 51:516-520.

Burman, J.O., Pontér, C., and Boström, K., 1978. Metaborate digestion procedure for inductively coupled plasma-optical emission spectrometry. Anal. Chem., 50:679-680.

Crow, E.L., Davis, F.A., and Maxfield, M.W., 1960. Statistics Manual: New York (Dover).

Dick, H.J.B., Erzinger, J., Stokking, L.B., et al., 1992. Proc. ODP, Init. Repts., 140: College Station, TX (Ocean Drilling Program).

Fairbairn, H.W., 1951. A cooperative investigation of precision and accuracy in chemical, spectrochemical and modal analysis of silicate rocks. U.S. Geol. Surv. Bull., 980.

Flanagan, F.J., 1986. Reference samples in geology and geochemistry. Geostand. Newsl., 10:191-264.

\footnotetext{
Abbreviations for names of organizations and publications in ODP reference lists follow the style given in Chemical Abstracts Service Source Index (published by American Chemical Society).
} 
Gladney, E.S., and Roelandts, I., 1988. 1987 compilation of elemental concentration data for USGS BHVO-1, MAG-1, QLO-1, RGM-1, SCo-1, SDC-1, SGR-1 and STM-1. Geostand. Newsl., 12:253-262.

Govindaraju, K., 1984. 1984 compilation of working values and sample description for 170 international reference samples of mainly silicate rocks and minerals. Geostand. Newsl., 8 (spec. iss).

Govindaraju, K., 1989a. 1988 compilation report on trace elements in six ANRT rock reference samples: Diorite DR-N, Serpentinite UB-N, Bauxite BX-N, Disthene DT-N, Granite GS-N and Potash feldspar FK-N. Geostand. Newsl., 13:5-67.

Govindaraju, K., 1989b. 1989 compilation of working values and sample description for 272 geostandards. Geostand. Newsl., 13 (spec. iss.).

Govindaraju, K. (Ed.), 1987. 1987 compilation on report on Ailsa Craig Granite AC-E with the participation of 128 GIT-IWG laboratories. Geostand, Newsl., 11:203-255.

Hergt, J.M., and Sims, Jr., D.R., 1994. Assessment of the precision of Leg 135 Shipboard XRF analyses and the contamination introduced by crushing in tungsten carbide. In Hawkins, J., Parson, L., Allan, J., et al., Proc. ODP, Sci. Results, 135: College Station, TX (Ocean Drilling Program), 925-929.

Horlick, G., and Shao, Y., 1992. Inductively coupled plasma-mass spectrometry for elemental analysis. In Montaser, A., and Golightly, D.W. (Eds.), Inductively Coupled Plasmas in Analytical Atomic Spectrometry (2nd ed.): New York (VCH Publ.), 551-612.

Hunt, D.T.E., and Wilson, A.L., 1986. The Chemical Analyses of Water: Cambridge, UK (Royal Society of Chemistry).

Lister, B., 1982. Evaluation of analytical data: a practical guide for geoanalysts. Geostand. Newsl., 6:175-205.

Lister, B., 1985. Looking at analytical data. Geostand. Newsl., 9:263-273.

Massart, D.L., Vandeginste, B.G.M., Deming, S.N., Michotte, Y., and Kaufman, L., 1988. Chemometrics: A Textbook: Amsterdam (Elsevier).

Mermet, J.M., 1992. Drift in inductively coupled plasma spectrochemistry: origins, diagnostics and correction methods [paper presented at Winter Conference on Plasma Spectrochemistry, San Diego, Jan. 6-11, 1992.], 55. (Abstract)

Montaser, A., 1992. Assessment of the potential and limitations of plasma sources compared to ICP discharges for analytical spectroscopy. In Montaser, A., and Golightly, D.W. (Eds.), Inductively Coupled Plasmas in Analytical Atomic Spectrometry (2nd ed.): New York (VCH Publ.), 1-52.

Moore, G.L., 1989. Introduction to Inductively Coupled Plasma Atomic Emission Spectrometry: Amsterdam (Elsevier).

Normand, M.D., Leeman, W.P., Blanchard, D.P., Fitton, J.G., and James, D., 1989. Comparison of major and trace element analyses by ICP, XRF, INAA and ID methods. Geostand. Newsl., 13:283-290.
Rona, P.A., Boström, K., and Epstein, S., 1980. Hydrothermal quartz vug from the Mid-Atlantic Ridge. Geology, 8:569-572.

Shipboard Scientific Party, 1992a. Explanatory notes. In Dziewonski, A., Wilkens, R., Firth, J., et al., 1992. Proc. ODP, Init. Repts., 136: College Station, TX (Ocean Drilling Program), 9-26.

, 1992b. Site 504. In Becker, K., Foss, G., et al., 1992. Proc. ODP, Init. Repts., 137: College Station, TX (Ocean Drilling Program), 15-55.

, 1992c. Site 828. In Collot, J.-Y., Greene, H.G., Stokking, L.B., et al., Proc. ODP, Init. Repts., 134: College Station, TX (Ocean Drilling Program), 139-177.

, 1992d. Site 829. In Collot, J.-Y., Greene, H.G., Stokking, L.B., et al., Proc. ODP, Init. Repts., 134: College Station, TX (Ocean Drilling Program), 179-260.

Sims, K.W., Gladney, E.S., Lundstrom, C., and Bower, N.W., 1988. Elemental concentrations in Japanese silicate rock standards: a comparison with the literature. Geostand. Newsl., 12:379-389.

Skoog, D.A., and West, D.M., 1982. Fundamentals of Analytical Chemistry: Philadelphia, (Saunders).

Snedecor, G.W., and Cochran, W.G., 1967. Statistical Methods (6th ed.): Ames, IA (Iowa State Col. Press).

Storms, M.A., Batiza, R., et al., 1993. Proc. ODP, Init. Repts., 142: College Station, TX (Ocean Drilling Program).

Thompson, M., and Walsh, J.N., 1983. A Handbook of Inductively Coupled Plasma Spectrometry: Glasgow (Blackie).

Verma, S.P., Besch, T., Guevara, M., and Dobrich, B.S., 1992. Determination of twelve trace elements in twenty-seven and ten major elements in twenty-three geochemical reference samples by X-ray fluorescence spectrometry, Geostand. Newsl., 16:301-309.

Walsh, J.N., 1980. The simultaneous determination of the major, minor and trace constituents of silicate rocks using inductively coupled plasma spectrometry. Spectrochim. Acta, Part B, 35:107-111.

Welz, B., 1985. Atomic Absorption Spectrometry (2nd ed.): Weinheim (VHC Verlagsgesell.).

Willard, H.H., Merritt, L.L., Jr., Dean, J.A., and Settle, F.A., Jr., 1988. Instrumental Methods of Analysis (7th ed.): Belmont, CA (Wadsworthe Publ.).

Date of initial receipt: 28 June 1993

Date of acceptance: 11 May 1994

Ms 142SR-112 